\title{
Position of parotid papilla and commissure of lip in relation to maxillary occlusal plane among patients visiting a tertiary care centre of Nepal
}

\author{
Mehta $\mathrm{P}^{1}$, Chhetri $\mathrm{S}^{2}$ \\ ${ }_{1,2}^{1,2}$ ecturer, Department of Prosthodontics and Maxillofacial Prosthetics, Nobel Medical College and Hospital, Biratnagar, \\ Nepal
}

\begin{abstract}
Introduction: Exact position of Parotid papilla and commissure of lip plays crucial role in determining maxillary occlusal plane which directly facilitates in rehabilitating edentulous patients with removable complete denture. Since the beginning many authors have conducted different studies regarding its position but multiple variations in its location have been noted. Also this type of study has not been conducted in Nepalese population which drags urge to determine the position of these soft tissue landmarks in context to Nepalese population.

Objective: To determine the position of parotid papilla and commissure of lip in relation to maxillary occlusal plane.

Materials and Methods: A descriptive cross-sectional study was conducted in the 45 dentate subjects visiting Nobel medical college from November 2019 to April 2020. A vestibular impression was made, stone index fabricated and vernier caliper was used to locate position of parotid papilla \& commissure of lip. All the data were collected and statistically analyzed.

Results: The mean measurement of distances between maxillary occlusal plane and parotid papilla was $3.69 \pm 1.19 \mathrm{~mm}$ and between maxillary occlusal plane and commissure of lip was $0.41 \pm 0.83 \mathrm{~mm}$ respectively.

Conclusion: This study showed that position of parotid papilla was superior to the position of maxillary occlusal plane with the mean distance of $3.69 \mathrm{~mm}$. Commissure of lip was found to be at the level of maxillary occlusion plane in highest number of cases followed by superior position with least at inferior position with respect to maxillary occlusal plane.
\end{abstract}

Key words: Dental occlusion, Commissures, Parotid Papilla

\section{Introduction}

$\mathrm{T}$ he determination of the occlusal plane is one of the most important clinical procedures during prosthodontic rehabilitation of edentulous patients. Incorrect record of the occlusal plane hampers esthetics, phonetics and mastication. It may affect the stability

\section{Conflict of Interest: No}

\section{*Corresponding Author}

Dr. Pawan Mehta, Lecturer

Department of Prosthodontics and Maxillofacial

Prosthetics, Nobel Medical College and Hospital,

Biratnagar, Nepal

E-mail: pawanmehta129@gmail.com of a complete denture and ultimately result in alveolar bone resorption. The position of occlusal plane in denture wearers should be as close as possible to the plane, which was previously occupied by the natural teeth. ${ }^{1}$ Such position of the occlusal plane provides normal function of the tongue and cheek muscles, thus enhancing the denture stability. It is believed that teeth oriented on an occlusal plane in harmony with the individuals physiognomy is responsible, in part, all conditions being equal, for stable, retentive full dentures. ${ }^{2}$

Among various landmarks, commonly used intraoral landmarks are parotid papilla and 
commissure of lip. According to Winkler, ${ }^{3}$ the parotid papilla is located $1 / 4$ th inch $(6 \mathrm{~mm})$ above the occlusal surface of maxillary first molar teeth. Lunquist and Luther ${ }^{4}$ measured the distance with cusp tip of maxillary molars as the reference for occlusal plane and suggested parotid papilla was located $4 \mathrm{~mm}$ above the occlusal plane. In a study done by Shigli et al, ${ }^{5}$ mean distance of parotid papilla was $2.56 \mathrm{~mm}$ above the maxillary occlusal plane. In another study conducted by Foley and Latta, ${ }^{6}$ edentulous patients were examined to determine the distance of occlusal plane from the inferior border of the right and left parotid papillae, using the parotid papilla as a reference guide for occlusal plane orientation. They found that the parotid papilla was $3.3 \mathrm{~mm}$ above the occlusal plane.

It is evident that various studies reported in literature allow variation in location parotid papilla and commissure of lip with respect to maxillary occlusal plane. Further, these measurements in various literature was basically done in Americans, ${ }^{4}$ Irani,${ }^{7}$ Jordanee, ${ }^{8}$ Indian ${ }^{9}$ population only. Hence there is a need to determine the position of parotid papilla and commissure of lip in context to Nepalese population.

\section{Materials and Methods}

This study was conducted in Department of Prosthodontics and Maxillofacial Prosthetics, Nobel Medical College and Teaching Hospital. Ethical approval for the study was obtained from Institutional Review Board, NMCTH. The duration of study was six months. This was a cross sectional observational study.

Purposive sampling was done among patients attending Nobel Medical College and Teaching Hospital. General screening was done on target population and subjects fulfilling the inclusion criteria were selected from them. This study considers $95 \% \mathrm{CI}$ and $80 \%$ power to estimate the sample size. In this regard the study considers mean and SD of parotid papilla and commissure of lip (i.e. $-6.16 \pm 3.91$ and $-5.64 \pm 3.94$ respectively), using the following formula:

$\mathrm{SD}=\mathrm{SD} 1+\mathrm{SD} 2 / 2, \mathrm{Z} \alpha=1.96$ at $95 \% \mathrm{CI}, \mathrm{Z} \beta=0.842$ at $80 \%$ power.

According to previous record of the department, only 50 cases had been reported in the last six months. Therefore, corrected sample size formula was used to estimate the sample size. Hence the actual sample size was 45 for this study.

Inclusion criteria included subjects with symmetrical alignment of maxillary dentition, Angle's class I maxillo-mandibular relationship, maxillary central incisors and the mesio-palatal cusp of the maxillary first molars contacting a flat occlusal plane indicator, absence of any apparent defect, deformity or asymmetry of face and subjects who agree for voluntary participation in the study. Exclusion criteria included subjects with tooth agenesis, crowding, spacing, rotation, retained teeth, supernumerary dentition, midline diastema, gross attrition, history of orthodontic, prosthodontic, restorative rehabilitation, periodontal disease, tooth mobility, history of periodontal surgery and congenital or acquired maxillary defects.

The materials used were: Irreversible hydrocolloid impression material (Zelgan, Dentsply), type III dental stone (Gypstone, Type III, PREVESTDenPro), self cure acrylic Resin: RR, Dentsply, India (Batch no: R130205) and modeling wax: orchid modeling wax, India. (Batch no: PY0312).

Selection of subjects: For all the selected participants, informed consent was taken after purposive sampling. It was made clear to them that the decision to participate in the study was completely voluntary and participation or denial will have no effect on their status as a patient. All the procedures to be done were explained 
in detail showing the instruments and materials to be used on them. They were also assured that the procedures were painless, harmless and completely free of cost.

\section{Making of upper and lower Impressions and Pouring of Casts}

Selection of appropriate size perforated stock metal tray was done for each subject for the maxillary \& mandibular arches. Irreversible hydrocolloid material (Zelgan, Dentsply) was mixed as per manufacturer's instruction. The material was loaded in previously selected maxillary \& mandibular arch trays and impressions were made.

\section{Fabrication of Maxillary and Mandibular Casts}

The maxillary and mandibular arch impressions were disinfected with $2 \%$ gluteraldehyde. As per manufacturer's instruction, Type III dental stone and water was mixed in vacuum mixing jar, mixed for 30 seconds and impressions were poured within 10 minutes. All the casts were retrieved within 30 to 45 minutes. This procedure was done for all participants to obtain 45 maxillary \& mandibular casts.

\section{Relationship of the maxillary occlusal plane to the parotid papilla and commissure of lips} Vestibular screen with perforation was fabricated on the stone cast (Figure 1). Vestibular impression (Figure 2) was made with Irreversible hydrocolloid impression material. The position of the parotid papilla (Figure.3) and commissure of lip (Figure 4) of the subject were marked with an indelible pencil and transferred to the irreversible hydrocolloid vestibular impression (Figure 5). Vestibular impression was trimmed to fit it again on the stone cast at maximum intercuspation (Figure 6). A stone index was then poured over the vestibular impression to obtain the record of the parotid papilla and commissure of the lips (Figure 7). After setting of stone index, vestibular impression and cast were removed (Figure 8,9).

Maxillary cast was placed on the surveyor with occlusal plane parallel to the floor and the stone index was positioned on the base of the maxillary cast. Maxillary occlusal plane was transferred in stone index (Figure 10). Level of parotid papilla and commissure of lip was measured in relation to maxillary occlusal plane in stone index with the digital vernier caliper (Figure 11).

\section{Data collection \& statistical analysis}

Data was entered in Microsoft Excel Sheet version 8.0. The data was cleaned and coded. It was then transferred to Statistical Package of Social Sciences (SPSS) version 17.0 for further analysis. The data was displayed using pie charts and frequency distribution tables. Continuous variables were summarized using mean, standard deviation and range. The level of significance was set at $5 \%$.

\section{Results}

The level of lip commissure with reference to the maxillary occlusal plane was observed. It was found that in majority (48.89\%) of the participants, the commissure of lip was at the level of occlusal plane and similar proportion $(42.22 \%)$ was above the occlusal plane while very few $(8.89 \%)$ was below the occlusal plane. (chart 1)

Table 1 describes all the continuous variables related to measurement of the current study. The mean measurement of distances between maxillary occlusal plane and parotid papilla is $3.69 \pm 1.19 \mathrm{~mm}$ and between maxillary occlusal plane and commissure of lip is $0.41 \pm 0.83 \mathrm{~mm}$ respectively. 
Table 1: Descriptive statistics for distances between maxillary occlusal plane and other landmarks.

\begin{tabular}{|c|c|c|c|c|}
\hline \multirow{2}{*}{ Landmarks } & \multirow{2}{*}{ Mean (mm) } & \multirow{2}{*}{ Standard Deviation } & \multicolumn{2}{c|}{ Range } \\
\cline { 4 - 5 } & & & Minimum & Maximum \\
\hline Parotid Papillae & 3.69 & 1.19 & 2.91 & 4.36 \\
\hline Commissure of the lip & 0.41 & 0.83 & 0.00 & 0.97 \\
\hline
\end{tabular}

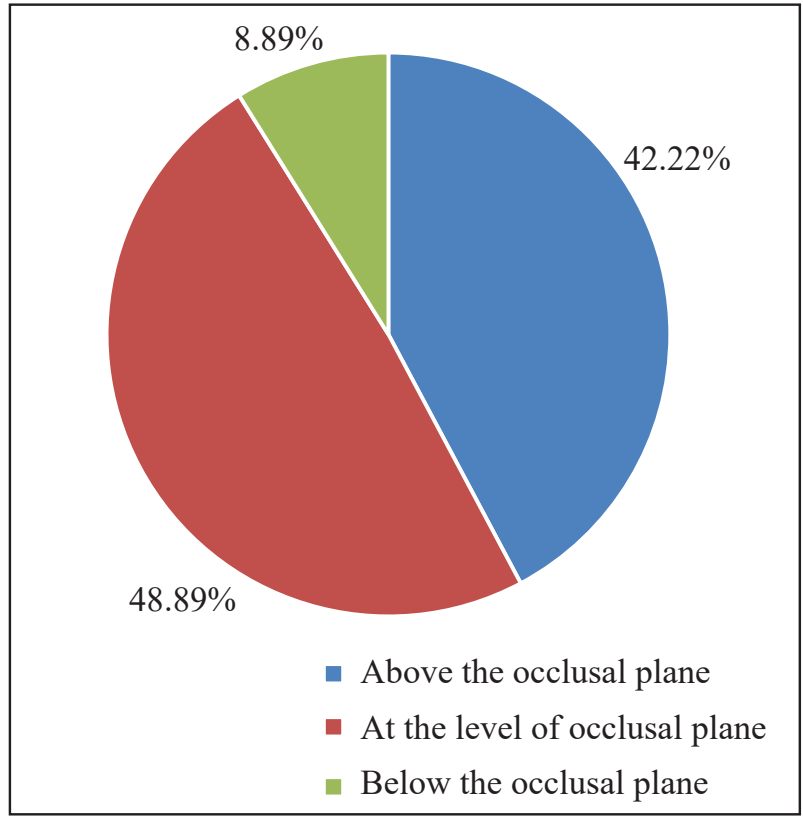

Chart 1: Position of lip commissure at different levels of maxillary occlusal plane $(n=45)$.

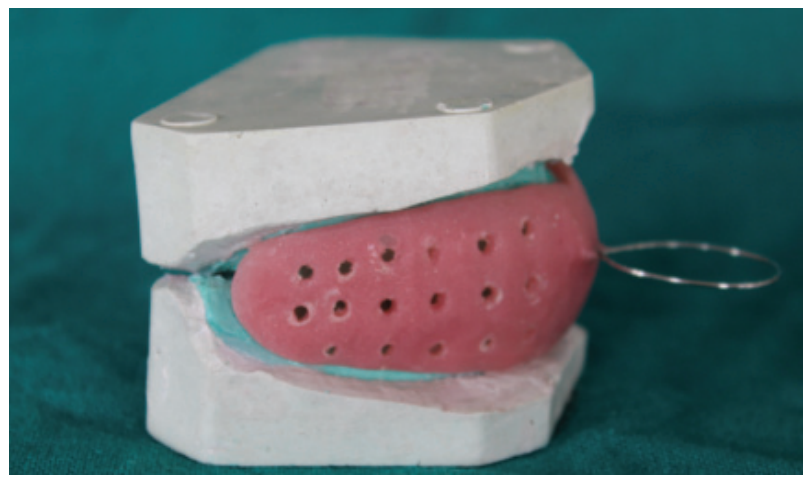

Figure 1: vestibular screen

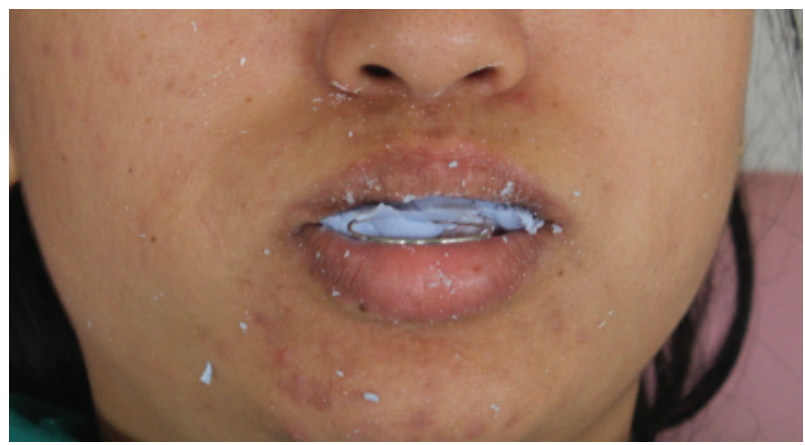

Figure 2: Making vestibular impression

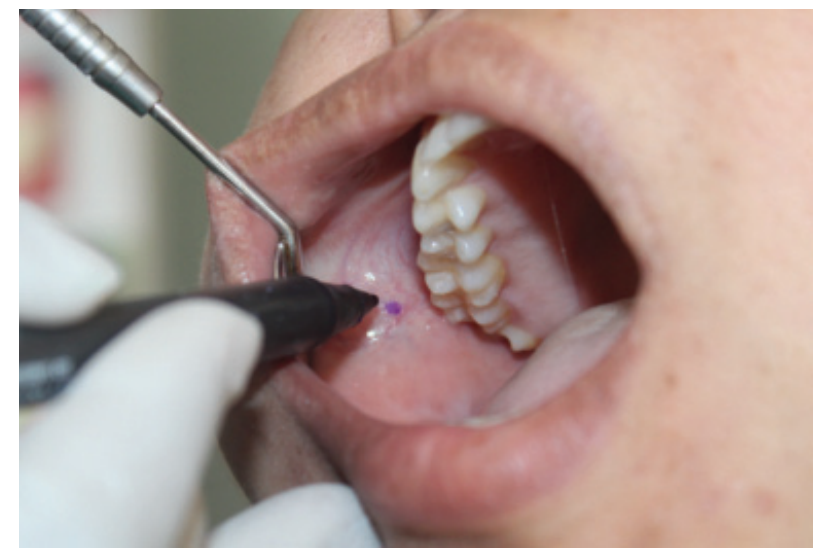

Figure 3: Marking parotid papilla

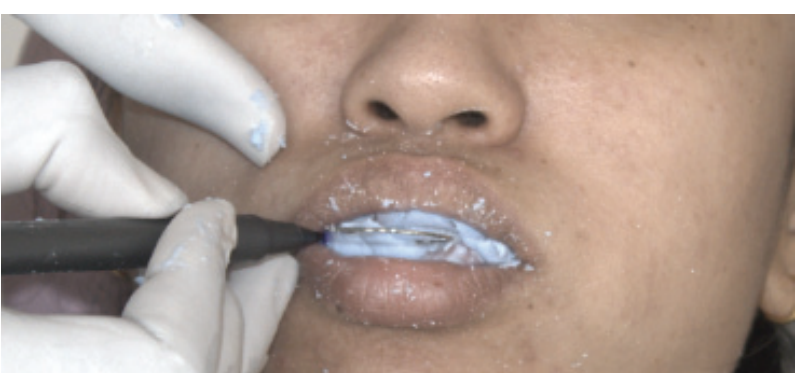

Figure 4: Marking commissure of lip

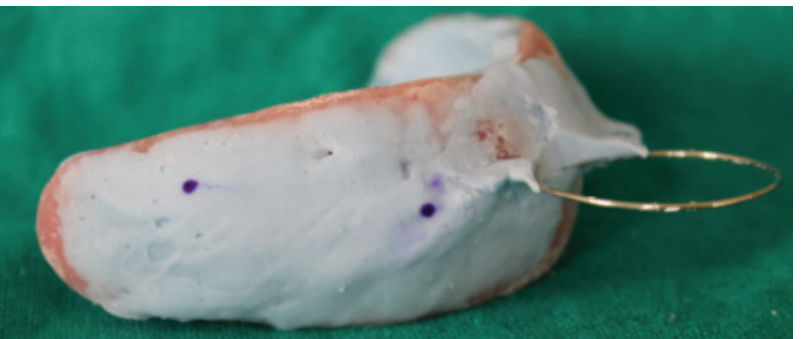

Figure 5: Marking transferred to vestibular impression

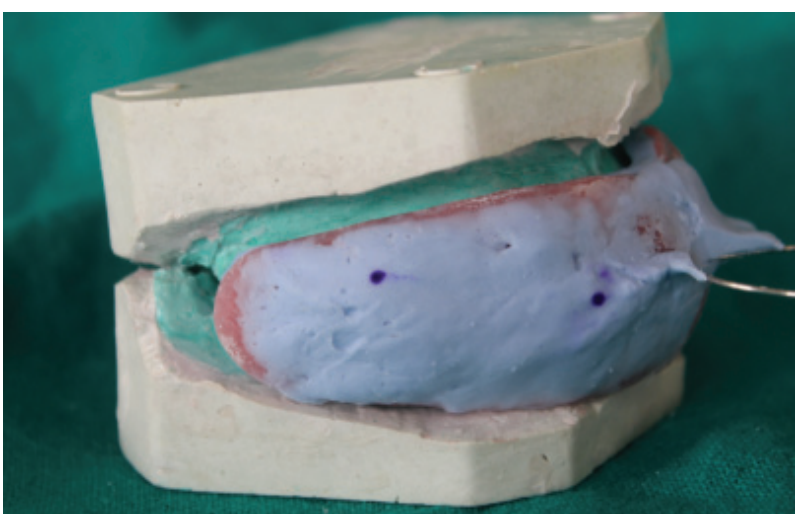

Figure 6: Fitting of vestibular impression at maximum intercuspation 


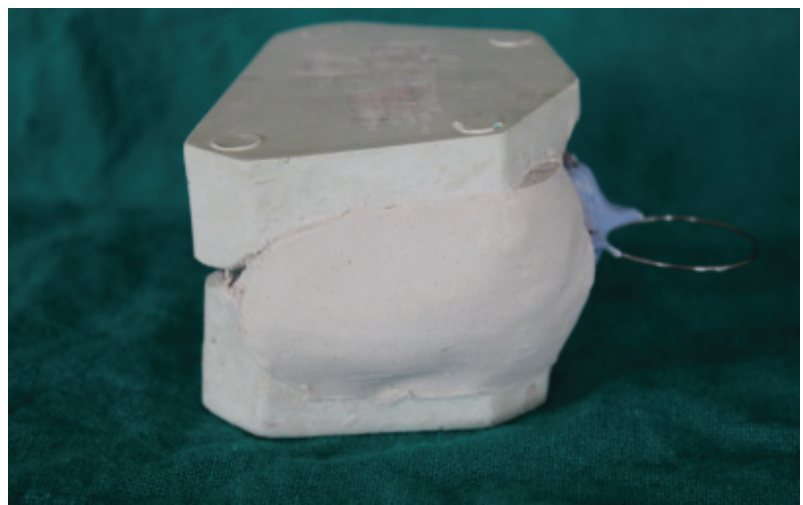

Figure 7: Making stone index

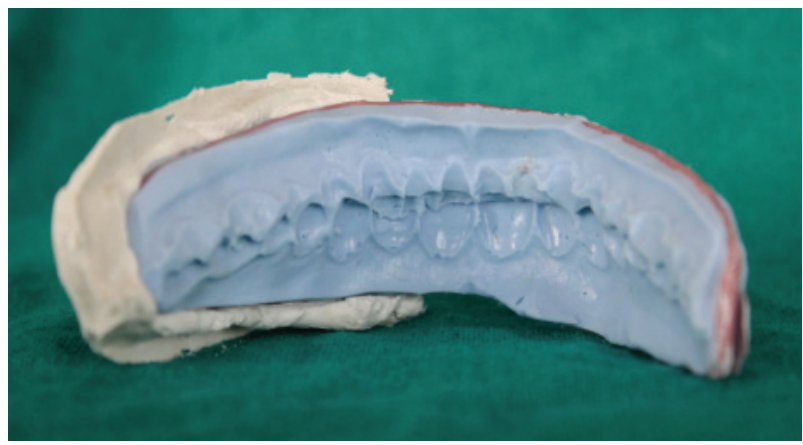

Figure 8: Removal of stone index along with vestibular impression after setting

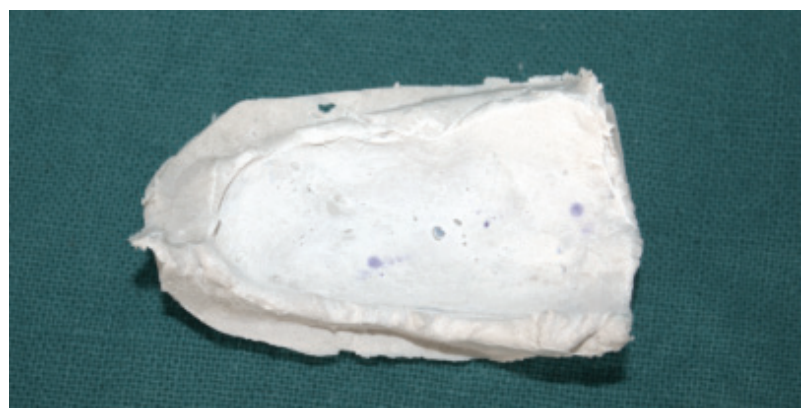

Figure 9: Marking obtained in stone index

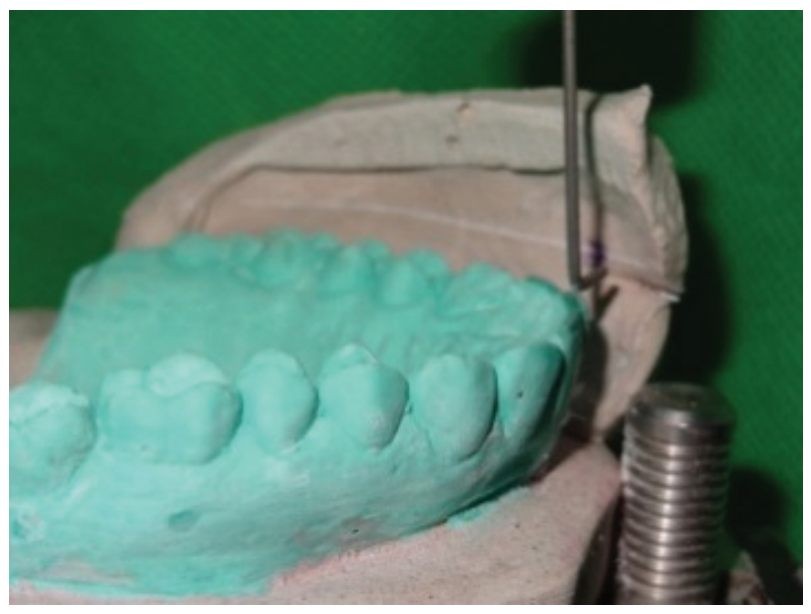

Figure 10: Fitting of vestibular impression at maximum intercuspation

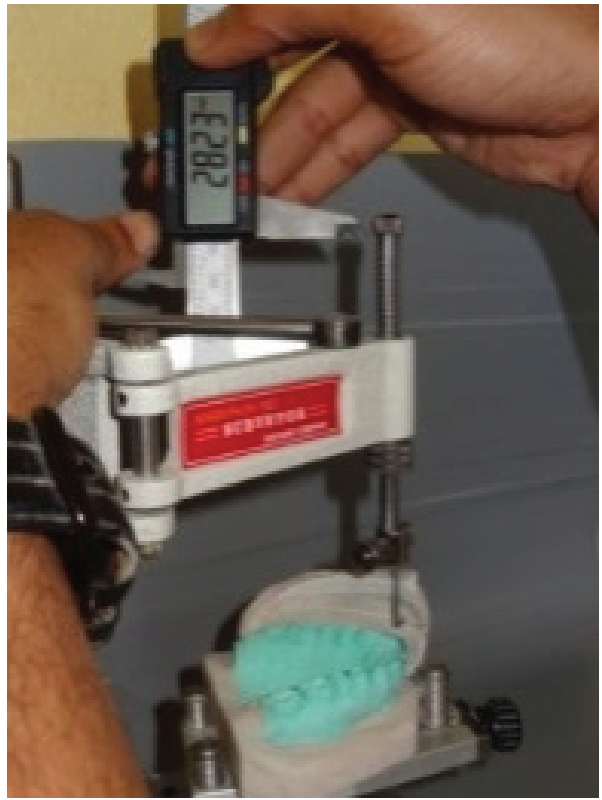

Figure 11: Measuring position of lip commissure $\&$ parotid papilla with digital vernier calliper

\section{Discussion}

Determining occlusal plane in edentulous patients poses a challenge to the clinician. In this situation, the occlusal plane should be relocated in a position in which it had been previously situated. It is because the stomatognathic system functions normally at this position and will continue functioning normally till it is altered. The position of the occlusal plane forms the basis for ideal teeth arrangement and should fulfill the necessary mechanical, esthetic, and phonetic requirements. ${ }^{10}$

Parotid papilla and commissure of lip are important landmarks and they may act as useful guide in determining the level of occlusion plane. These landmarks had not been investigated scientifically and only few studies had been done regarding the validity of these landmarks. Therefore, this study was done to assess the position of parotid papilla and commissure of lip for determination of occlusal plane.

For determining the position of parotid papilla and commissure of lip, vestibular impression method was used because of its simplicity and easy availability. Parotid papilla as intraoral 
land mark acts as useful guide in determining the occlusal plane. In this study, the mean measurement of distances between maxillary occlusal plane and parotid papilla is $3.69 \pm 1.19$ $\mathrm{mm}$, which is in agreement with the Foley \& Latta study and Shigli study whereas disagrees with Winklers study ${ }^{3}$ which suggested that the parotid papilla is located $1 / 4$ th inch $(6 \mathrm{~mm})$ above the occlusal surface of maxillary first molar teeth.

Besides the parotid papilla, commissure of lip also has a great role in determining level of occlusal plane. The commissure of lip is a dynamic landmark when compared to other stationary anatomical landmarks such as the retromolar pad and ala-tragus line. ${ }^{11,12}$ Within the limits of this study, the authors tried to observe the level of lip commissure with reference to the maxillary occlusal plane. It was found that commissure of lip was at the level occlusal plane in $48.89 \%$, above the occlusal plane in $42.22 \%$ and below the occlusal plane in $8.89 \%$. Thus, the commissure of lip appears to be useful landmark in physiologic and esthetic orientation of the occlusal plane.

Though this study has been conducted, the limitation of this study is the participants were between 18 and 30 years old, whereas the results would be applicable to edentulous patients who are usually older. Age changes within parotid papilla and commissure of lips were not taken into consideration. Therefore, further study in larger group of subjects is needed to verify the validity of this method to be used during fabrication of complete dentures.

\section{Conclusion}

Parotid papilla was found to be superior to the position of maxillary occlusion plane with the mean distance of $3.69 \mathrm{~mm}$. Commissure of lip was found to be at the level of maxillary occlusal plane in $48.89 \%$, superior to maxillary occlusal plane in $42.22 \%$ and inferior to maxillary occlusal plane in $8.89 \%$.

\section{References}

1. Čelebic A., et al. A study of the occlusal plane orientation by intra-oral method (retromolar pad). J Oral Rehabil 1995; 22(3):-233-6.

2. MG, S., Complete dentures, 2 nd edn. The C.V. Mosby Company. St. Louis 1947-180.

3. Winkler S Ishiyaku Euro America Inc., S.L. Essentials of complete denture prosthodontics,2nd edn. (2004).

4. Lundquist, D.O. and W.W. Luther. Occlusal plane determination. J Prosthet Dent 1970; 23(5):489-98.

5. Shigli, K., B. Chetal, and J. Jabade. Validity of soft tissue landmarks in determining the occlusal plane. The Journal of Indian Prosthodontic Society 2005; 5:3-139.

6. Foley, P. and G. Latta. A study of the position of the parotid papilla relative to the occlusal plane. J Prosthet Dent 1985; 53:1- 124.

7. Sadr, K. and M. Sadr. A study of parallelism of the occlusal plane and ala-tragus line. Journal of dental research, dental clinics, dental prospects 2010; 3:4-107.

8. Quran, F.A.A., A. Hazza'a, and N.A. Nahass. The position of the occlusal plane in natural and artificial dentitions as related to other craniofacial planes. Journal of Prosthodontics 2010; 19:8-601.

9. Gupta, R., H. Aeran, and S. Singh. Relationship of anatomic landmarks with occlusal plane. The Journal of Indian Prosthodontic Society 2009; 9:3-142.

10. Kumar, D., et al. Guidelines in the Arrangement of Lower Incisor Teeth-Review and Clinical Implications. International Journal of Contemporary Dentistry 2011;2:5.

11. Fish and E.W. Using the muscles to stabilize the full lower denture. The Journal of the American Dental Association 1922; 20:12- 2163.

12. Beresin, V.E. and F.J. Schiesser. The neutral zone in complete dentures. J Prosthet Dent 1976; 36:4-356. 